\title{
Quadriceps Strength and Osteoarthritis Progression in Malaligned and Lax Knees
}

Leena Sharma, MD; Dorothy D. Dunlop, PhD; September Cahue, BS; Jing Song, MS; and Karen W. Hayes, PhD

Background: Quadriceps muscle strengthening is a common goal in the management of knee osteoarthritis. In healthy knees, strength protects against new osteoarthritis. In arthritic knees, greater strength may protect joints and thereby delay osteoarthritis progression. Alternatively, in certain joint environments, such as malalignment or laxity, greater strength may translate into damaging joint reaction forces. The relationship between quadriceps strength and progression of knee osteoarthritis may differ according to these factors.

Objective: To determine whether greater quadriceps strength is associated with greater probability of tibiofemoral osteoarthritis progression in malaligned knees and in high-laxity knees.

Design: Prospective, longitudinal cohort study.

Setting: Academic medical center.

Participants: 237 persons with primary knee osteoarthritis, definite tibiofemoral osteophytes, and at least some difficulty with knee-requiring activity. Two hundred thirty completed the 18month evaluation. The current study primarily involved those without advanced osteoarthritis in either knee $(n=171)$.

Measurements: Quadriceps strength, knee laxity and alignment, and osteoarthritis progression.
Results: The predicted probability of tibiofemoral progression was $0.153(95 \% \mathrm{Cl}, 0.100$ to 0.228$)$ in high-strength knees and $0.098(\mathrm{Cl}, 0.061$ to 0.155$)$ in low-strength knees. In malaligned knees, high strength was associated with a significant increase $(P=0.03)$ in the likelihood of progression (predicted probability, $0.406[\mathrm{Cl}, 0.226$ to 0.615$]$ vs. $0.187[\mathrm{Cl}, 0.081$ to 0.375$]$ in high-strength vs. low-strength knees). Strength was also associated with increased likelihood of progression in high-laxity knees ( $P=0.003$ when high laxity was defined as $\geq 6.75$ degrees). The probability of patellofemoral progression did not differ between high- and low-strength knees in the full sample or within subsets.

Conclusions: Greater quadriceps strength at baseline was associated with increased likelihood of tibiofemoral osteoarthritis progression in malaligned knees and lax knees. Subset-specific approaches beyond strengthening exercises should be developed to enhance joint-protective muscle activity.

Ann Intern Med. 2003;138:613-619.

www.annals.org

For author affiliations, see end of text.

See editorial comment on pp 678-679.
$\mathbf{K}^{\mathrm{n}}$ nee osteoarthritis is responsible for more chronic disability in elderly persons than any other medical condition (1). Quadriceps strengthening is widely recommended for knee osteoarthritis $(2,3)$, based on cross-sectional studies identifying strength (maximal voluntary muscle force generation) as a correlate of physical function $(4-8)$ and on trials, predominantly short term, suggesting that quadriceps strengthening reduces pain and improves function $(9,10)$.

The impact of quadriceps strength on the course of osteoarthritic disease itself is not well understood. A longitudinal study has shown that, in healthy knees, strong quadriceps offer some protection against new osteoarthritis development (11). However, whether quadriceps strength protects against progression or advancement of osteoarthritis in already arthritic knees has not been demonstrated. Brandt and colleagues (12) found no difference in baseline quadriceps strength between those with and those without disease progression. The effect of quadriceps strength on osteoarthritis disease progression is particularly important given the frequency with which quadriceps strengthening exercises are prescribed for persons with knee osteoarthritis.

Muscle effects are less predictable in arthritic knees than in healthy knees. On the positive side, muscle activity promotes cartilage health and stabilizes the joint. During activity, muscles contract at different levels and protective reflexes are applied to shield knee tissues from injury (13). Coactivation, the dual drive of agonist and antagonist muscles, provides control for the stop and start of motion as well as compensation for gravity. Implicit in the recommendation of quadriceps strengthening for knee osteoarthritis is the assumption that greater strength will enhance these positive effects. If this enhancement comes without cost, greater strength may indeed protect arthritic joints from osteoarthritis progression.

On the negative side, greater quadriceps strength may be associated with forces that could damage the vulnerable articular cartilage of osteoarthritis. The compensatory increase in muscle forces in osteoarthritis may increase the joint reaction force (14). A higher coactivation level in the agonist versus antagonist muscles can impair motion regulation and reduce ligament-protecting actions (13). The net impact of quadriceps strength on osteoarthritis progression depends on which effects-positive or negative-are greater.

Which muscle effects prevail is likely to depend on the local mechanical environment. Local factors that alter load distribution, such as laxity and malalignment, influence how well the joint copes with muscle forces. Woo and associates (15) liken this situation to a hammer (muscle) driving a nail (the joint), while a hand (ligaments and, more broadly, local environment) holds the nail in place. 
ARTICLE Quadriceps Strength and Osteoarthritis Progression in Malaligned and Lax Knees

\section{Context}

Experts routinely recommend that adults with knee osteoarthritis strengthen leg muscles.

\section{Contribution}

This 18-month cohort study of 237 adults with primary knee osteoarthritis found that greater baseline quadriceps strength was associated with greater risk for progressive tibiofemoral joint space narrowing among adults with malaligned or very lax knees.

\section{Implications}

Maximization of quadriceps strength in osteoarthritic patients with malaligned or very lax knees may not be joint protective and should be studied in trials that include joint structure outcomes.

\section{Cautions}

These results do not imply that physical activity in adults with knee osteoarthritis is harmful.

The stabilizing hand allows greater force from the hammer. In other words, a healthy environment contributes to safe muscle force distribution over the menisci, articular cartilage, and other tissues. However, with laxity or malalignment, muscle forces may increase stress on localized areas of cartilage. Similarly, Marks and colleagues (16) theorized that local joint abnormalities can render muscle forces pathogenic.

Malalignment and laxity are key local abnormalities. Any shift from a neutral hip-knee-ankle alignment alters load distribution; varus and valgus alignments increase medial and lateral compartment forces, respectively (17). Alignment influences the outcome of most knee surgeries, as well as natural disease progression and functional decline in osteoarthritis (18). Knee laxity-abnormal displacement of the tibia with respect to the femur (19)—shifts opposing surfaces of tibiofemoral contact so that congruence is reduced and increases shear and compression forces. Varusvalgus laxity has been linked to greater likelihood of osteoarthritis after ligament injury $(20,21)$. In other studies, laxity was present in patients with osteoarthritis before fullblown disease and was worsened by aspects of disease (22), was associated with worse function, and altered the strength-function relationship (23).

If greater quadriceps strength improves the load imbalance created by malalignment or attenuated load in malaligned knees, it might protect against osteoarthritis progression in patients with maligned knees. Alternatively, malalignment may alter the line of action of quadriceps forces, distributing them inequitably across the joint surface (16); in this case, strength might increase the likelihood of osteoarthritis progression. In the lax knee, strength might be protective if greater quadriceps strength enhances

dynamic stabilization. However, if the cost of this compensation-that is, greater joint reaction force-exceeds the stabilization benefit, the likelihood of osteoarthritis progression might increase. Our objective was to determine whether greater quadriceps strength was associated with greater probability of tibiofemoral osteoarthritis progression among all knees in persons with osteoarthritis and in two subsets, malaligned knees and high-laxity knees.

\section{Methods \\ Participants}

Mechanical Factors in Arthritis of the Knee (MAK) is a natural history study of knee osteoarthritis at Northwestern University in Chicago, Illinois. Participants in MAK were recruited through periodicals targeting senior citizens, 67 neighborhood organizations, the registry of the Northwestern University Buehler Center on Aging, and medical center referrals. Inclusion and exclusion criteria were based on those developed for osteoarthritis progression studies at a workshop sponsored by the National Institute of Arthritis and Musculoskeletal and Skin Diseases and the National Institute on Aging (24). Inclusion criteria were definite osteophyte presence (Kellgren and Lawrence radiographic grade $\geq 2$ ) in one or both knees and at least a little difficulty (Likert category) with at least two items in the Western Ontario and McMaster University osteoarthritis index physical function scale. Exclusion criteria were corticosteroid injection within 3 months, avascular necrosis, rheumatoid or other inflammatory arthritis, periarticular fracture, Paget disease, villonodular synovitis, joint infection, ochronosis, neuropathic arthropathy, acromegaly, hemochromatosis, Wilson disease, osteochondromatosis, gout, pseudogout, osteopetrosis, bilateral total knee replacement, or plan for knee replacement within the next year. Persons with past unilateral knee replacement were eligible if they had osteoarthritis in the nonreplaced knee. The institutional review board of Northwestern University approved the study, and all participants gave informed consent.

\section{Measurement of Key Factors}

All measurements were obtained in both knees. Isokinetic quadriceps strength was tested by using a computerdriven isokinetic dynamometer (Cybex, Avocent, Huntsville, Alabama) to assess maximal torque during movement. One tester assessed all participants by following a previously described protocol (23). The computer recorded data in foot-pounds (ft-lbs) and corrected for gravity effects. Reliability, determined by using test repetitions, was high (intraclass correlation coefficients $>0.98)(23)$.

To assess alignment, a single anteroposterior radiograph of both lower extremities was obtained by using a graduated grid cassette $(51 \times 14$ inches), adhering to a protocol we have described elsewhere (18). Alignment was defined as the measure of the angle formed by the intersection of the line connecting the centers of the femoral 
Quadriceps Strength and Osteoarthritis Progression in Malaligned and Lax Knees ARTICLE

head and intercondylar notch and the line connecting the centers of the ankle talus and tibial spines $(17,25,26)$. Knees were considered "more neutral" if the angle was less than 5 degrees in a varus or valgus direction and "malaligned" if the angle was 5 degrees or more. One experienced reader made all measurements. Reliability, based on a set of radiographs from patients with osteoarthritis (18), was high for varus and valgus alignment (intraclass correlation coefficients, 0.99 and 0.98 , respectively).

Varus-valgus laxity was measured with a device designed for the MAK study by Dr. Thomas Buchanan (22, $23)$. The measurement protocol has been described elsewhere $(22,23)$. Angular deviation was measured at the foot with application of varus or valgus load. Varus-valgus laxity was analyzed as the sum of varus and valgus rotation for each knee (27-29). Because a definition of high and low laxity is not available, knees in the highest laxity tertile ( $\geq 5.75$ degrees) were designated as high laxity. Laxity measurements were performed by the same examiner and assistant, and reliability was very good in participants with osteoarthritis (within-session intraclass correlation coefficients, 0.85 to 0.96 ; between-sessions intraclass correlation coefficients, 0.84 to 0.90 ) (22).

\section{Measurement of Outcome}

Knee radiographs were obtained at baseline and at 18 months, following the semi-flexed, fluoroscopically confirmed protocol developed by Buckland-Wright (24, 30, 31 ). Knee position, beam alignment, markers to account for magnification, and measurement landmarks were specified. The standing semi-flexed position superimposes anterior and posterior medial tibial margins. Knee position was confirmed fluoroscopically before anteroposterior radiographs were taken. To visualize the patellofemoral compartment, weight-bearing, 30-degree flexion, skyline views were obtained (30). Radiographs were obtained in one unit by two trained technicians. Foot maps made at baseline were used at the 18-month evaluation.

We used a consistent approach to define osteoarthritis progression in each compartment, relying on joint space assessment. The primary outcome, tibiofemoral osteoarthritis progression, was defined as an increase in the grade of joint space narrowing in the medial or lateral compartment between baseline and 18 months. Patellofemoral osteoarthritis progression was defined as an increase in the grade of medial or lateral patellofemoral narrowing. The semi-flexed, fluoroscopically confirmed protocol provides appropriate conditions for direct measurement of medial but not lateral or patellofemoral joint space width; no protocol has yet been developed to support lateral or patellofemoral joint space measurement. One experienced reader graded each compartment separately by using the Osteoarthritis Research Society International atlas-based scales (none, possible, definite, severe) developed by Altman and colleagues (32). Intrareader reliability was good $(\kappa=0.80$ to 0.86$)$ (18).

\section{Measurement of Potential Confounders}

Body mass index (BMI) was measured as weight in kilograms divided by the square of the height in meters. Disease severity was assessed by using the Kellgren and Lawrence global scale $(0=$ normal; $1=$ possible osteophytes; 2 = definite osteophytes, possible joint space narrowing; $3=$ moderate or multiple osteophytes, definite narrowing, some sclerosis, possible attrition; $4=$ large osteophytes, marked narrowing, severe sclerosis, definite attrition). Physical activity was assessed by using the Physical Activity Scale for the Elderly (PASE) (33).

\section{Statistical Analysis}

Participants with advanced osteoarthritis (the most severe grade of joint space narrowing at baseline in any compartment of either knee) were excluded from analysis. Replaced knees were excluded from analyses. We used logistic regression with generalized estimating equations (GENMOD procedure, SAS software, version 8.00, SAS Institute, Inc., Cary, North Carolina) to include data from one or both knees of each participant and thereby test the effect of high versus low strength on likelihood of osteoarthritis progression. An independent working correlation structure was used. Low strength was defined as less than or equal to the median strength of right lower limbs in the sample $(47.3 \mathrm{ft}-\mathrm{lb})$. Statistical tests on logistic regression coefficients for strength were based on robust empirical standard error estimates. Analyses were adjusted for potential confounders: age (continuous), BMI (continuous), disease severity using Kellgren and Lawrence grade (categories entered as indicator variables), and physical activity using PASE score (continuous). To illustrate the effect of high versus low strength, we presented the predicted probability of osteoarthritis progression and associated 95\% CIs for a person 65 years of age who had a baseline BMI of 30 $\mathrm{kg} / \mathrm{m}^{2}$, a Kellgren and Lawrence grade of 2, and a median PASE score of 124, based on the estimated logistic model. The relationship between strength and progression was examined in subsets of more neutral, malaligned, low-laxity, and high-laxity knees. Analyses were repeated by using alternative definitions of high laxity.

\section{Role of the Funding Sources}

The funding sources had no role in the collection, analysis, and interpretation of the data or in the decision to submit the paper for publication.

\section{RESULTS}

We longitudinally followed 237 participants, of whom 7 (3\%) did not return at 18 months; 5 had died, and 2 could not be reached. Of the 230 remaining participants, 171 did not have advanced osteoarthritis in any compartment of either knee. Among these 171 participants (126 women, $45 \mathrm{men})$, mean age $( \pm \mathrm{SD})$ was $64.0 \pm 11.0$ years and mean BMI $( \pm S D)$ was $30.0 \pm 5.5 \mathrm{~kg} / \mathrm{m}^{2}$. At baseline, no participants had a Kellgren and Lawrence grade of 0 for

\footnotetext{
15 April 2003 Annals of Internal Medicine $\mid$ Volume 138 - Number $8 \mid \mathbf{6 1 5}$
} 
ARTICLE $\mid$ Quadriceps Strength and Osteoarthritis Progression in Malaligned and Lax Knees

Table 1. Knees with Tibiofemoral Osteoarthritis Progression according to Baseline Alignment and Strength

\begin{tabular}{|lll}
\hline Variable & Knees, $\boldsymbol{n}$ & $\begin{array}{l}\text { Knees with } \\
\text { Osteoarthritis } \\
\text { Progression, \% }\end{array}$ \\
$\begin{array}{l}\text { More neutral alignment ( }<5 \text { degrees) } \\
\text { Low quadriceps strength }\end{array}$ & 125 & 11.2 \\
$\quad$ High quadriceps strength & 125 & 11.2 \\
Malalignment ( $\geq 5$ degrees)† & & \\
Low quadriceps strength & 38 & 26.3 \\
High quadriceps strength & 40 & 50.0 \\
\hline
\end{tabular}

* 147 participants.

† 59 participants.

right knee osteoarthritis severity, 11 had a grade of 1,110 had a grade of 2, 50 had a grade of 3, and none had a grade of 4. Sixty-three participants had no joint space narrowing in the right knee at baseline, 60 had mild narrowing, 48 had moderate narrowing, and none had severe narrowing. Mean right-limb quadriceps strength $( \pm S D)$ was $51.8 \pm$ $28.5 \mathrm{ft}-\mathrm{lb}$, and mean laxity $( \pm S D)$ was $5.32 \pm 2.03 \mathrm{de}-$ grees. Right knees were varus in 79 participants, valgus in 71 participants, and neutral in 21 participants. No participants had a knee effusion. These assessments were similar in the left knees. All analyses incorporated data from both knees, using generalized estimating equations. We excluded 14 knees that had previously been replaced, leaving 328 knees for analysis.

Quadriceps strength did not reduce the likelihood of subsequent osteoarthritis progression. After adjustment for age, BMI, disease severity, and physical activity, the predicted probability of progression was slightly greater in knees with higher compared with lower quadriceps strength $(0.153$ [ $95 \% \mathrm{CI}, 0.100$ to 0.228$]$ vs. 0.098 [CI, 0.061 to 0.155$] ; P=0.09$ ).

We determined the proportion of knees with disease progression according to baseline alignment and strength (Table 1). In more neutral knees, quadriceps strength had no effect on progression $(11.2 \%$ in high- and low-strength knees). However, disease progression was substantially more likely in high-strength versus low-strength malaligned knees (50.0\% vs. $26.3 \%)$. We next determined the proportion of knees with disease progression according to

Table 2. Knees with Tibiofemoral Osteoarthritis Progression according to Baseline Laxity and Strength

\begin{tabular}{|lll}
\hline Variable & Knees, $\boldsymbol{n}$ & $\begin{array}{l}\text { Knees with } \\
\text { Osteoarthritis } \\
\text { Progression, \% }\end{array}$ \\
$\begin{array}{c}\text { Low laxity (<5.75 degrees)* } \\
\text { Low quadriceps strength }\end{array}$ & 98 & 14.3 \\
High quadriceps strength & 120 & 19.2 \\
High laxity ( $\geq 5.75$ degrees) & & \\
Low quadriceps strength & 65 & 15.4 \\
High quadriceps strength & 45 & 24.4 \\
\hline
\end{tabular}

* 127 participants.

+71 participants.

616 15 April 2003 Annals of Internal Medicine $\mid$ Volume $138 \cdot$ Number 8 baseline laxity and strength (Table 2). High strength versus low strength at baseline was associated with increased progression in low-laxity $(19.2 \%$ vs. $14.3 \%)$ and high-laxity (24.4\% vs. $15.4 \%)$ knees.

We used logistic regression to statistically test the relationship between strength and tibiofemoral progression in the alignment (Table 3) and laxity (Tables 4 and 5) subsets. Predicted probabilities are provided to illustrate these results. There was no evidence of a protective effect of strength on progression in any knee subset. In contrast, in malaligned knees (Table 3), strength was associated with a significant increase in the likelihood of disease progression. As shown in Table 4, strength in high-laxity knees was associated with an increased likelihood of progression that approached significance. When the cutoff for high laxity was increased to at least 6.75 degrees (Table 5), the association became significant.

The predicted probability of patellofemoral progression did not differ between high- and low-strength knees, either in the full sample or within knee subsets. Knee pain during strength testing was infrequent, and adjustment for pain did not alter our results. Strength, alignment, laxity, BMI, and physical activity were also measured at 18 months. Except for laxity, which increased by 1.8 degrees, mean change in each of these factors was small. Modest correlations existed between baseline values of strength and laxity $(r=-0.29)$, alignment and laxity $(r=0.18)$, and strength and physical activity $(r=0.29)$ but were not of sufficient magnitude to influence interpretation of results.

When analyses were repeated in women only, results were similar in high-laxity knees. For high versus low strength, the $P$ value was 0.03 when high laxity was defined as at least 5.75 degrees and 0.001 when high laxity was defined as at least 6.75 degrees. Results were also similar in malaligned knees but were not statistically significant, possibly reflecting the small number of knees in this subset. Because our study included much fewer men than women, it was not feasible to confirm results in men only.

Our sample had a high average BMI. Therefore, it was important to determine whether our results persisted in nonobese participants. In participants with BMIs less than $30 \mathrm{~kg} / \mathrm{m}^{2}(n=117)$, progression was more likely in highstrength malaligned knees $(P=0.06)$ and high-strength, high-laxity knees $(P=0.03$ when high laxity was defined as $\geq 5.75$ degrees; $P=0.05$ when high laxity was defined as $\geq 6.75$ degrees) than in low-strength knees.

\section{DIsCUSSION}

Greater quadriceps strength at baseline did not protect against subsequent progression of knee osteoarthritis and, in malaligned knees and in lax knees, was associated with increased likelihood of tibiofemoral osteoarthritis progression. Strength was not associated with increased or decreased likelihood of osteoarthritis progression in more neutrally aligned or low-laxity knees. These results suggest 
Quadriceps Strength and Osteoarthritis Progression in Malaligned and Lax Knees ARTICLE

Table 3. Predicted Probability of Osteoarthritis Progression for High versus Low Strength according to Baseline Alignment

\begin{tabular}{lllr}
\hline Baseline Alignment & $\begin{array}{l}\text { Participants/Knees, } \\
n / n\end{array}$ & \multicolumn{2}{c}{$\begin{array}{c}\text { Predicted Probability of Osteoarthritis Progression Adjusted for Age, Body Mass } \\
\text { Index, Disease Severity, and Physical Activity* }\end{array}$} \\
\cline { 3 - 4 } & & High Strength & Low Strength \\
More neutral ( $<5$ degrees) & $147 / 250$ & $0.079(0.045-0.136)$ & $0.090(0.050-0.155)$ \\
Malaligned ( $\geq 5$ degrees) & $59 / 78$ & $0.406(0.226-0.615)$ & $0.187(0.081-0.375)$ \\
\hline
\end{tabular}

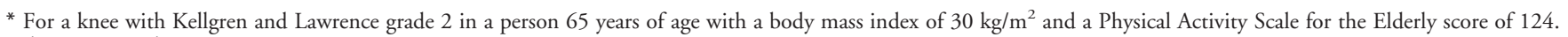
Values in parentheses are $95 \%$ CIs.

+ Obtained from logistic regression testing in which the quadriceps strength effect equaled zero.

that the positive actions of the quadriceps muscle on knee function are not translated by greater strength into a beneficial change in disease course.

Our findings are consistent with those of Brandt and colleagues (12), who reported no difference in baseline quadriceps strength between persons with progressive versus nonprogressive tibiofemoral osteoarthritis over an average of 32 months. In a study of incident osteoarthritis, quadriceps strength protected against new knee osteoarthritis in women (11). In contrast to these findings for the knee, Chaisson and colleagues (34) found that grip strength was associated with an increased risk for incident osteoarthritis at the metacarpophalangeal joints in women and the proximal interphalangeal joints, metacarpophalangeal joints, and thumb base in men.

Together, the studies of Brandt and colleagues (11, 12) and Chaisson and colleagues (34) show that the effect of strength may differ between joint sites and according to baseline joint status, that is, whether the joint is relatively healthy (as in studies of incident osteoarthritis) or arthritic (as in studies of osteoarthritis progression). Our results demonstrate that the relationship between strength and progression can differ within a joint site according to attributes of the local environment. Muscle forces that are beneficial in the nondiseased knee may have adverse effects in the more vulnerable osteoarthritic knee that neutralize the benefits of strength or, in some knee subsets, have a net negative effect.

In knees with low laxity or more neutral alignment, strength was not associated with an increased or decreased likelihood of progression, suggesting a balance between positive and negative consequences of strength. Our findings in malaligned knees support Marks and colleagues' theory (16) that such knees are less able to evenly distribute muscle forces. In high-laxity knees, our findings support that muscle-related dynamic stabilization increases the joint reaction force (14). In terms of the natural history of osteoarthritis, our results indicate that strength does not mechanically compensate for the ill effects of malalignment or laxity on disease outcome.

We examined the relationship between strength at baseline and change in osteoarthritic disease over an 18month period. The only other longitudinal report of the strength-progression relationship also examined strength at baseline (12). A next step, more feasible within a strengthening intervention trial, would be to examine the effect of an increase in strength on subsequent disease progression. This was not possible in our study because strength was examined at baseline and progression, between baseline and 18 months. It is difficult, however, to believe that a strength increase could delay disease progression in malaligned or lax knees when greater baseline strength was associated with a worse outcome.

Limitations of our study include the fact that our sample was not population based. However, participants were recruited from a variety of community sources as well as a medical center and are likely to be comparable to persons in the general population who receive widespread recommendations on quadriceps strengthening from providers and the lay literature. To date, laxity and full-limb alignment have not been described in population-based studies of knee osteoarthritis. It is unclear what constitutes high versus low quadriceps strength in persons with established mild to moderate knee osteoarthritis; in this report, we applied the sample median. Analyses using alternative definitions of high strength yielded similar results. The sex distribution of our sample allowed us to confirm findings separately in women, but not in men.

Table 4. Predicted Probability of Osteoarthritis Progression for High versus Low Strength according to Laxity at Baseline

\begin{tabular}{|lllr}
\hline Laxity at Baseline & $\begin{array}{c}\text { Participants/Knees, } \\
n / n\end{array}$ & \multicolumn{2}{c}{$\begin{array}{c}\text { Predicted Probability of Osteoarthritis Progression Adjusted for Age, Body Mass } \\
\text { Index, Disease Severity, and Physical Activity* }\end{array}$} \\
\cline { 3 - 4 } & & High Strength & Low Strength \\
Low laxity $(<5.75$ degrees) & $127 / 218$ & $0.124(0.068-0.218)$ & $0.103(0.057-0.179)$ \\
High laxity $(\geq 5.75$ degrees) & $71 / 110$ & $0.178(0.078-0.357)$ & $0.074(0.032-0.164)$ \\
\hline
\end{tabular}

* For a knee with Kellgren and Lawrence grade 2 in a person 65 years of age with a body mass index of $30 \mathrm{~kg} / \mathrm{m}^{2}$ and Physical Activity Scale for the Elderly score of 124 . Values in parentheses are $95 \%$ CIs.

† Obtained from logistic regression testing in which the quadriceps strength effect equaled zero. 
ARTICLE Quadriceps Strength and Osteoarthritis Progression in Malaligned and Lax Knees

Table 5. Predicted Probabilities of Osteoarthritis Progression for High versus Low Strength for High-Laxity Knees with Alternative Definitions of High Laxity

\begin{tabular}{|lllc}
\hline $\begin{array}{l}\text { Definition of High } \\
\text { Laxity at Baseline }\end{array}$ & $\begin{array}{l}\text { Participants/Knees, } \\
n / n\end{array}$ & \multicolumn{1}{c}{$\begin{array}{c}\text { Predicted Probability of Osteoarthritis Progression Adjusted for Age, Body Mass } \\
\text { Index, Disease Severity, and Physical Activity* }\end{array}$} \\
\cline { 3 - 3 } & & High Strength & Low Strength \\
$\geq 4.75$ degrees & $104 / 168$ & $0.158(0.086-0.273)$ & $0.099(0.052-0.181)$ \\
$\geq 5.75$ degrees & $71 / 110$ & $0.178(0.078-0.357)$ & $0.074(0.032-0.179)$ \\
$\geq 6.25$ degrees & $60 / 91$ & $0.176(0.065-0.395)$ & $0.069(0.024-0.181)$ \\
$\geq 6.75$ degrees & $49 / 70$ & $0.307(0.101-0.637)$ & $0.041(0.009-0.168)$
\end{tabular}

* For a knee with Kellgren and Lawrence grade 2 in a person 65 years of age with a body mass index of $30 \mathrm{~kg} / \mathrm{m}^{2}$ and a Physical Activity Scale for the Elderly score of 124 . Values in parentheses are $95 \%$ CIs.

† Obtained from logistic regression testing in which the quadriceps strength effect equaled zero.

Strengthening exercise is recommended to reduce pain and improve physical function in knee osteoarthritis, but there is minimal information on its long-term impact (3). Numerous short-term trials demonstrate benefits of exercise; however, when restricted to strengthening exercise exclusively, effect sizes were small $(9,10)$. In another trial with 18-month follow-up, Ettinger and colleagues found that strengthening exercise led to modest improvement in function (35). There are several possible reasons for a smaller than expected effect in short- and long-term studies. Strength may be less important than aerobic capacity or psychosocial factors in long-term function. Osteoarthritis-associated impairments, such as laxity, may limit the impact of strength on function. Also, strength may have a negative effect on osteoarthritis disease course in certain knee subsets, which ultimately reduces its beneficial effect on function.

Our results raise questions about the use of quadriceps strengthening. First, they question the assumption that greater strength enhances muscle activity in a way that benefits disease course or causes no harm in all patients with osteoarthritis. Second, our results suggest that strength maximization is not likely to have a beneficial disease-modifying effect in persons with osteoarthritis and malaligned or lax knees. Third, the relationship between strength and osteoarthritis progression hinges on local environment and may not be reducible to a simple tenet or strengthening prescription. Fourth, concomitantly dealing with the negative effects of muscle forces may make strengthening exercise more effective as a strategy to prevent disability. Our results have implications for quadriceps strengthening in knee osteoarthritis but not for other forms of exercise or physical activity.

Because trends in osteoarthritis management favor generic, minimally supervised exercise, our findings are timely. The availability of resistance exercise equipment in fitness clubs creates opportunities for additional unsupervised strengthening exercise and contributes to assumptions that such exercise is without risk and that "more" must be better. These assumptions may lead to knee exercises performed under high load. In the future, clinical trials that include a strengthening intervention should ex- amine the impact of a strength increase on joint structure within alignment and laxity subsets. Strength maintenance programs tailored to knee subsets should be developed.

Muscle-enhancing interventions, rather than exercises focused solely on strengthening, should also be further developed. Such interventions might directly target specific joint-protective muscle actions while safely maintaining strength, possibly through supervised, balanced agonistantagonist (quadriceps-hamstring) exercise; exercise that targets separate components of the quadriceps; exercises that improve muscle endurance; gait training; exercise that enhances quadriceps action during common tasks; and exercise that improves proprioceptive accuracy. Future studies should also develop approaches coupling muscle enhancement with other interventions that improve load distribution and stabilize the knee. Such approaches may better capitalize on the beneficial effects of strong muscles while neutralizing the deleterious effects of muscle forces, thereby delaying osteoarthritis progression. In our study, greater quadriceps strength at baseline was associated with increased likelihood of tibiofemoral osteoarthritis progression in malaligned knees and in lax knees. These results support the need to develop subset-specific approaches to enhance joint-protective muscle activity.

From Northwestern University, Chicago, Illinois.

Grant Support: By National Institutes of Health grant AR-30692, National Institutes of Health/National Center for Research Resources grant RR-00048, and the Arthritis Foundation.

Potential Financial Conflicts of Interest: None disclosed.

Requests for Single Reprints: Leena Sharma, MD, Division of Rheumatology, Feinberg School of Medicine, Northwestern University Medical School, 300 East Superior Avenue, Tarry Building 3-715, Chicago, IL 60611.

Current author addresses and author contributions are available at www .annals.org. 


\section{References}

1. Guccione AA, Felson DT, Anderson JJ, Anthony JM, Zhang Y, Wilson PW, et al. The effects of specific medical conditions on the functional limitations of elders in the Framingham Study. Am J Public Health. 1994;84:351-8. [PMID: 8129049]

2. Recommendations for the medical management of osteoarthritis of the hip and knee: 2000 update. American College of Rheumatology Subcommittee on Osteoarthritis Guidelines. Arthritis Rheum. 2000;43:1905-15. [PMID: 11014340] 3. Hicks JE, Perry MB, Gerber LH. Rehabilitation in the management of patients with osteoarthritis. In: Moskowitz RW, Howell DS, Altman RD, Buckwalter JA, Goldberg VM, eds. Osteoarthritis, Diagnosis and Medical/Surgical Management. Philadelphia: WB. Saunders; 2001:413-46.

4. McAlindon TE, Cooper C, Kirwan JR, Dieppe PA. Determinants of disability in osteoarthritis of the knee. Ann Rheum Dis. 1993;52:258-62. [PMID: 8484690]

5. Rejeski WJ, Craven T, Ettinger WH Jr, McFarlane M, Shumaker S. Selfefficacy and pain in disability with osteoarthritis of the knee. J Gerontol B Psychol Sci Soc Sci. 1996;51:P24-9. [PMID: 8548514]

6. Lankhorst GJ, Van de Stadt RJ, Van der Korst JK. The relationships of functional capacity, pain, and isometric and isokinetic torque in osteoarthrosis of the knee. Scand J Rehabil Med. 1985;17:167-72. [PMID: 4081669]

7. van Baar ME, Dekker J, Lemmens JA, Oostendorp RA, Bijlsma JW. Pain and disability in patients with osteoarthritis of hip or knee: the relationship with articular, kinesiological, and psychological characteristics. J Rheumatol. 1998;25: 125-33. [PMID: 9458215]

8. Fisher NM, Pendergast DR, Gresham GE, Calkins E. Muscle rehabilitation: its effect on muscular and functional performance of patients with knee osteoarthritis. Arch Phys Med Rehabil. 1991;72:367-74. [PMID: 2059102]

9. van Baar ME, Assendelft WJ, Dekker J, Oostendorp RA, Bijlsma JW. Effectiveness of exercise therapy in patients with osteoarthritis of the hip or knee: a systematic review of randomized clinical trials. Arthritis Rheum. 1999;42:1361-9. [PMID: 10403263]

10. Baker K, McAlindon T. Exercise for knee osteoarthritis. Curr Opin Rheumatol. 2000;12:456-63. [PMID: 10990187]

11. Slemenda C, Heilman DK, Brandt KD, Katz BP, Mazzuca SA, Braunstein $\mathrm{EM}$, et al. Reduced quadriceps strength relative to body weight: a risk factor for knee osteoarthritis in women? Arthritis Rheum. 1998;41:1951-9. [PMID: 9811049]

12. Brandt KD, Heilman DK, Slemenda C, Katz BP, Mazzuca SA, Braunstein EM, et al. Quadriceps strength in women with radiographically progressive osteoarthritis of the knee and those with stable radiographic changes. J Rheumatol. 1999;26:2431-7. [PMID: 10555906]

13. Solomonow M, D’Ambrosia R. Neural reflex arcs and muscle control of knee stability and motion. In: Scott WN, ed. The Knee. St. Louis: Mosby; 1994:107-20.

14. Schipplein OD, Andriacchi TP. Interaction between active and passive knee stabilizers during level walking. J Orthop Res. 1991;9:113-9. [PMID: 1984041] 15. Woo SL, Fenwick JA, Kanamori A, Gil JE, Chan Saw SS, Vogrin TM. Biomechanical consideration of joint function. In: Moskowitz RW, Howell DS, Altman RD, Buckwalter JA, Goldberg VM, eds. Osteoarthritis, Diagnosis and Medical/Surgical Management. Philadelphia: WB Saunders; 2001:145-69.

16. Marks R, Percy JS, Semple J, Kumar S. Quadriceps femoris activation changes in genu varum: a possible biomechanical factor in the pathogenesis of osteoarthrosis. J Theor Biol. 1994;170:283-9. [PMID: 7996856]

17. Tetsworth K, Paley D. Malalignment and degenerative arthropathy. Orthop Clin North Am. 1994;25:367-77. [PMID: 8028880]
18. Sharma L, Song J, Felson DT, Cahue S, Shamiyeh E, Dunlop DD. The role of knee alignment in disease progression and functional decline in knee osteoarthritis. JAMA. 2001;286:188-95. [PMID: 11448282]

19. Markolf KL, Bargar WL, Shoemaker SC, Amstutz HC. The role of joint load in knee stability. J Bone Joint Surg Am. 1981;63:570-85. [PMID: 7217123] 20. Kannus P. Nonoperative treatment of grade II and III sprains of the lateral ligament compartment of the knee. Am J Sports Med. 1989;17:83-8. [PMID: 2929843]

21. Kannus P. Long-term results of conservatively treated medial collateral ligament injuries of the knee joint. Clin Orthop. 1988;(226):103-12. [PMID: 3335084]

22. Sharma L, Lou C, Felson DT, Dunlop DD, Kirwan-Mellis G, Hayes KW, et al. Laxity in healthy and osteoarthritic knees. Arthritis Rheum. 1999;42:86170. [PMID: 10323441]

23. Sharma L, Hayes KW, Felson DT, Buchanan TS, Kirwan-Mellis G, Lou C, et al. Does laxity alter the relationship between strength and physical function in knee osteoarthritis? Arthritis Rheum. 1999;42:25-32. [PMID: 9920010]

24. Dieppe P, Altman RD, Buckwalter JA, Felson DT, Hascall V, Lohmander LS, et al. Standardization of methods used to assess the progression of osteoarthritis of the hip or knee joints. In: Kuettner KE, Goldberg VM, eds. Osteoarthritic Disorders. Rosemont, IL: American Academy of Orthopaedic Surgeons; 1995:481-96.

25. Chao EY, Neluheni EV, Hsu RW, Paley D. Biomechanics of malalignment. Orthop Clin North Am. 1994;25:379-86. [PMID: 8028881]

26. Hsu RW, Himeno S, Coventry MB, Chao EY. Normal axial alignment of the lower extremity and load-bearing distribution at the knee. Clin Orthop. 1990;(255):215-27. [PMID: 2347155]

27. Brage ME, Draganich LF, Pottenger LA, Curran JJ. Knee laxity in symptomatic osteoarthritis. Clin Orthop. 1994;(304):184-9. [PMID: 8020213]

28. Pottenger LA, Phillips FM, Draganich LF. The effect of marginal osteophytes on reduction of varus-valgus instability in osteoarthritic knees. Arthritis Rheum. 1990;33:853-8. [PMID: 2363739]

29. Wada M, Imura S, Baba H, Shimada S. Knee laxity in patients with osteoarthritis and rheumatoid arthritis. Br J Rheumatol. 1996;35:560-3. [PMID: 8670577]

30. Buckland-Wright C. Protocols for precise radio-anatomical positioning of the tibiofemoral and patellofemoral compartments of the knee. Osteoarthritis Cartilage. 1995;3 Suppl A:71-80. [PMID: 8581753]

31. Altman R, Brandt K, Hochberg M, Moskowitz R, Bellamy N, Bloch DA, et al. Design and conduct of clinical trials in patients with osteoarthritis: recommendations from a task force of the Osteoarthritis Research Society. Results from a workshop. Osteoarthritis Cartilage. 1996;4:217-43. [PMID: 11048620]

32. Altman RD, Hochberg M, Murphy WA Jr, Wolfe F, Lequesne M. Atlas of individual radiographic features in osteoarthritis. Osteoarthritis Cartilage. 1995;3 Suppl A:3-70. [PMID: 8581752]

33. Washburn RA, Smith KW, Jette AM, Janney CA. The Physical Activity Scale for the Elderly (PASE): development and evaluation. J Clin Epidemiol. 1993;46:153-62. [PMID: 8437031]

34. Chaisson CE, Zhang Y, Sharma L, Kannel W, Felson DT. Grip strength and the risk of developing radiographic hand osteoarthritis: results from the Framingham Study. Arthritis Rheum. 1999;42:33-8. [PMID: 9920011]

35. Ettinger WH Jr, Burns R, Messier SP, Applegate W, Rejeski WJ, Morgan $\mathrm{T}$, et al. A randomized trial comparing aerobic exercise and resistance exercise with a health education program in older adults with knee osteoarthritis. The Fitness Arthritis and Seniors Trial (FAST). JAMA. 1997;277:25-31. [PMID: 8980206] 
Current Author Addresses: Dr. Sharma and Ms. Cahue: Department of Medicine, Feinberg School of Medicine, Northwestern University Medical School, 300 East Superior Avenue, Tarry Building 3-715, Chicago, IL 60611.

Dr. Dunlop and Ms. Song: Institute for Health Services Research and Policy Studies, Northwestern University, 339 East Chicago Avenue, Wieboldt Hall, Room 717, Chicago, IL 60611.

Dr. Hayes: Department of Physical Therapy and Human Movement Sciences, Northwestern University Medical School, 645 North Michigan Avenue, Suite 1100, Chicago, IL 60611.

Author Contributions: Conception and design: L. Sharma, S. Cahue, K.W. Hayes.
Analysis and interpretation of the data: L. Sharma, D.D. Dunlop, J. Song.

Drafting of the article: L. Sharma, S. Cahue.

Critical revision of the article for important intellectual content: L. Sharma, D.D. Dunlop, K.W. Hayes.

Final approval of the article: L. Sharma, D.D. Dunlop, K.W. Hayes. Provision of study materials or patients: S. Cahue.

Statistical expertise: D.D. Dunlop, J. Song.

Obtaining of funding: L. Sharma.

Administrative, technical, or logistic support: L. Sharma, J. Song, K.W. Hayes.

Collection and assembly of data: L. Sharma, S. Cahue, K.W. Hayes. 